\title{
Flavor distributions in the nucleons: SU(2) sea asymmetry or isospin symmetry breaking?
}

\author{
Bo-Qiang $\mathrm{Ma},{ }^{*}$ Andreas Schäfer, and Walter Greiner \\ Institut für Theoretische Physik der Universität Frankfurt am Main, Postfach 1119 32, D-6000 Frankfurt, Germany
}

(Received 27 August 1992)

\begin{abstract}
The Gottfried sum-rule violation reported by the New Muon Collaboration was interpreted as an indication for a flavor asymmetry of the sea quark in the nucleon. We investigate the alternative possibility that isospin symmetry between the proton and the neutron is breaking. We examine systematically the consequences of this possibility for several processes, namely, neutrino deep inelastic scattering, the charged pion Drell-Yan process, the proton Drell-Yan process, and semi-inclusive deep inelastic scattering, and conclude that a decision between the two alternative explanations is possible.
\end{abstract}

PACS number(s): 14.20.Dh, 13.15.-f, 13.60.Hb, 13.85.Qk

\section{INTRODUCTION}

Recently, the measurement of the Gottfried sum [1] by the New Muon Collaboration (NMC) [2] has inspired a number of investigations on the sea-quark flavor distributions of nucleons [3-21]. The Gottfried sum is defined, in terms of the proton and neutron structure functions $F_{2}(x)$, as $S_{G}=\int_{0}^{1}\left[F_{2}^{p}(x)-F_{2}^{n}(x)\right] d x / x$, which, when expressed in terms of quark momentum distributions $q_{i}^{N}(x)$ in the nucleon $N$, reads

$$
S_{G}=\int_{0}^{1} \sum_{i} e_{i}^{2}\left[q_{i}^{p}(x)+\bar{q}_{i}^{p}(x)-q_{i}^{n}(x)-\bar{q}_{i}^{n}(x)\right] d x
$$

where $e_{i}$ is the charge of a quark of flavor $i$. Using flavor conservation, isospin symmetry between the proton and the neutron, and further the flavor distribution symmetry in the sea, one arrives at the Gottfried sum rule (GSR) $S_{G}=1 / 3$. In the NMC experiment, the value of $S_{G}$ was determined from $F_{2}^{p}-F_{2}^{n}$ expressed as

$$
F_{2}^{p}-F_{2}^{n}=2 F_{2}^{D}\left(1-F_{2}^{n} / F_{2}^{p}\right) /\left(1+F_{2}^{n} / F_{2}^{p}\right),
$$

where the ratio $F_{2}^{n} / F_{2}^{p}=2 F_{2}^{D} / F_{2}^{p}-1$ was determined from the deuteron/proton cross-section ratio measured in the experiment. The data cover the kinematic range of $x=0.004-0.8$ for $Q^{2}=4 \mathrm{GeV}^{2}$. Assuming a smooth extrapolation of the data $F_{2}^{n} / F_{2}^{p}$ from $x=0.8$ to 1 , and adopting a Regge behavior $a x^{b}$ for $F_{2}^{p}-F_{2}^{n}$, a flavor nonsinglet, in the region $x=0.004-0.15$ and then extrapolating it to $x=0$, the NMC reported the value for the Gottfried sum

$$
S_{G}=0.240 \pm 0.016
$$

\footnotetext{
* On leave from Institute of High Energy Physics, Academia Sinica, P.O. Box 918(4), Beijing 100039, China.
}

which is significantly below the simple quark-partonmodel result of $1 / 3$.

Several different effects have been proposed as the source for this discrepancy between the NMC data and the GSR. In several early works [3-6] the NMC data were claimed as evidence for a flavor distribution asymmetry in the sea of nucleons, i.e., an excess of $d \bar{d}$ over $u \bar{u}$ pairs in the proton [3-6]. This suppression of $u \bar{u}$ pairs could be due to the Pauli exclusive principle and the excess of a $u$ valence quark in the proton $[22,9]$. A flavor-SU(2) asymmetry in the sea can also be attributed to the pionic contribution as an excess of $p \rightarrow n+\pi^{+}$over $p \rightarrow \Delta^{++}+\pi^{-}$ (or $\pi^{+}$over $\pi^{-}$) [4-8], or from a more microscopic point of view to the excess of $u \rightarrow d+\pi^{+}$over $u \rightarrow u+\pi^{0}$ and $d \rightarrow u+\pi^{-}$over $d \rightarrow d+\pi^{0}[10,11]$. Other possibilities are that the smaller NMC result for the GSR is due to the unjustified $x \rightarrow 0$ extrapolation of the available data [12] or due to a small admixture of vector diquarks in a particular quark-diquark model of the nucleon [13]. More recently, it has been observed [14] that the violation of the GSR could also be due to isospin symmetry breaking between the proton and the neutron, while the sea flavor distribution symmetry of nucleons is preserved. According to this explanation there are more sea quarks in the neutron than in the proton. Nuclear effects have also been examined [15-18], and it has been suggested that, e.g., the mesonic exchanges in the deuteron [15] could account for the difference between the NMC data and the GSR, although nuclear shadowing corrections may lead to smaller values of $S_{G}$ than reported by the NMC [17, 18].

In this paper we discuss the possibility of explicit isospin symmetry breaking between the proton and the neutron, i.e., $u^{p}(x) \neq d^{n}(x)$, etc. For simplicity we shall always assume sea flavor symmetry $\left[\bar{u}^{N}(x)=\bar{d}^{N}(x)\right]$ although both effects could occur simultaneously. We shall review the various experiments proposed to determine the origin of the small NMC data and analyze what results would be expected for our assumptions. In the paper by Kumano and Londergan [18] it was argued that a combination of neutrino structure functions of the pro- 
ton and deuteron, or an expression of the relative DrellYan cross sections for charged pions scattering from nuclear targets, are sensitive to the quantity $\bar{d}-\bar{u}$. Ellis and Stirling [19] suggested comparing $p p \rightarrow l^{+} l^{-} X$ and $p n \rightarrow l^{+} l^{-} X$ to distinguish between the $\mathrm{SU}(2)$ asymmetry in the sea and a non-Regge behavior at small $x$. Levelt, Mulders, and Schreiber [20] discussed the possible measurement of the asymmetry in the sea-quark distribution using semi-inclusive leptoproduction of charged pions and, more generally, by charged hadrons, to check for flavor asymmetries. These above processes were discussed before the observation [14] that isospin symmetry breaking between the proton and the neutron could be an alternative source for the GSR violation. The purpose of this paper is to examine the influences from $p$-n isospin symmetry breaking for the above processes. It is necessary to point out that the nuclear mesonic exchange explanation [15] also suggested an excess of mesonic contributions over the isospin symmetric $p-n$ in deuteron and thus should give the same results as isospin symmetry breaking if free neutrons are not involved in the measurements.

\section{DEEP INELASTIC NEUTRINO SCATTERING}

The possibility of distinguishing between an $\mathrm{SU}(2)$ sea asymmetry and $p$ - $n$ isospin symmetry breaking through deep inelastic neutrino and antineutrino scattering on protons and deuterons has been discussed in Ref. [14]. If the $\mathrm{SU}(2)$ sea is asymmetric the violation of the GSR indicates an excess of $d \bar{d}$ over $u \bar{u}$; i.e.,

$$
\int_{0}^{1}[\bar{u}(x)-\bar{d}(x)]=-0.140 \pm 0.024
$$

whereas for $p-n$ isospin symmetry breaking the violation of GSR is due to an excess of sea quarks in neutrons over those in protons while preserving the $\mathrm{SU}(2)$ symmetry in the sea of nucleons; i.e.,

$$
\int_{0}^{1}\left[\bar{q}^{p}(x)-\bar{q}^{n}(x)\right]=-0.084 \pm 0.014 .
$$

Both explanations are fitted to the observed $S_{G}$ but may give very different values for some linear combinations of neutrino structure functions from protons and deuterons. In Ref. [14] a new sum, defined by

$$
S=\int_{0}^{1}\left[\left(F_{2}^{\nu p}+F_{2}^{\bar{\nu} p}\right)-\frac{1}{2}\left(F_{2}^{\nu D}+F_{2}^{\bar{\nu} D}\right)\right] d x
$$

was suggested. This new sum is zero for an asymmetric sea explanation and $4 \int_{0}^{1}\left[\bar{q}^{p}(x)-\bar{q}^{n}(x)\right]=-0.336 \pm 0.058$ for $p-n$ symmetry breaking. Some other combinations, such as those indicated in Ref. [14], may also be chosen to distinguish between the two explanations.

The linear combination of neutrino structure functions from protons and deuterons,

$$
\frac{1}{2}\left[F_{2}^{\nu D}(x)-x F_{3}^{\nu D}(x)\right]-\left[F_{2}^{\nu P}(x)-x F_{3}^{\nu P}(x)\right],
$$

which was proposed by Kumano and Londergan [18] to measure the difference $\bar{u}-\bar{d}$, should be

$$
2 x[\bar{d}(x)-\bar{u}(x)]
$$

in the case of $\mathrm{SU}(2)$ asymmetry in the sea. This combination, when expressed in terms of quark momentum distributions, should read

$$
2 x\left[\bar{u}^{n}(x)-\bar{u}^{p}(x)\right],
$$

while it gives

$$
2 x\left[\bar{q}^{n}(x)-\bar{q}^{p}(x)\right]
$$

in the case of isospin symmetry breaking between the proton and the neutron. The two cases give different values for Eq. (2.4). From Eqs. (2.1) and (2.2), one can see that the prediction for Eq. (2.4) is less for the $n-p$ isospin symmetry breaking than for the $\mathrm{SU}(2)$ sea asymmetry.

\section{DRELL-YAN PROCESSES}

\section{A. Drell-Yan processes using charged pion from nuclear targets}

Also in Ref. [18] the quantity $\bar{d}(x)-\bar{u}(x)$ was suggested to be measured by Drell-Yan processes $\pi^{ \pm} A \rightarrow l^{+} l^{-} X$ initiated by charged pion scattering from nuclear targets. For large $x_{\pi}$, say $x_{\pi}>0.4$, the cross section per nucleon for $\pi^{+}$and $\pi^{-}$scattering from nuclear targets will be proportional to

$$
\begin{aligned}
& \tilde{\sigma}\left(\pi^{+} A\right)=V_{\pi}[ \frac{1}{2}\left(d^{n}+4 \bar{u}^{n}+d^{p}+4 \bar{u}^{p}\right) \\
&\left.+\varepsilon\left(d^{n}+4 \bar{u}^{n}-d^{p}-4 \bar{u}^{p}\right)\right], \\
& \tilde{\sigma}\left(\pi^{-} A\right)=V_{\pi}\left[\begin{array}{l}
\frac{1}{2}\left(4 u^{n}+\bar{d}^{n}+4 u^{p}+\bar{d}^{p}\right) \\
\left.+\varepsilon\left(4 u^{n}+\bar{d}^{n}-4 u^{p}-\bar{d}^{p}\right)\right],
\end{array}\right.
\end{aligned}
$$

where $\varepsilon \equiv N / A-1 / 2$ is the neutron excess of the target with $A$ nucleons and $V_{\pi}$ is the valence distribution of the pion. Thus one can define the ratio

$R_{\text {sea }}=\frac{4\left[\tilde{\sigma}\left(\pi^{+} A_{1}\right)-\tilde{\sigma}\left(\pi^{+} A_{0}\right)\right]+\tilde{\sigma}\left(\pi^{-} A_{1}\right)-\tilde{\sigma}\left(\pi^{-} A_{0}\right)}{\tilde{\sigma}\left(\pi^{+} A_{0}\right)-\tilde{\sigma}\left(\pi^{-} A_{0}\right)}$.

In the case of an asymmetric $\mathrm{SU}(2)$ sea, one assumes $p-n$ isospin symmetry: i.e.,

$$
\begin{aligned}
& u=u^{p} \leftrightarrow d^{n}, \quad d=d^{p} \leftrightarrow u^{n}, \\
& \bar{u}=\bar{u}^{p} \leftrightarrow \bar{d}^{n}, \quad \bar{d}=\bar{d}^{p} \leftrightarrow \bar{u}^{n} ;
\end{aligned}
$$

then one gets

$$
R_{\text {sea }}=\frac{10\left(\varepsilon_{1}-\varepsilon_{0}\right)(\bar{u}-\bar{d})}{u_{V}+d_{V}} .
$$

In the case of $p$ - $n$ isospin symmetry breaking, we assume that only the valence quarks preserve the isospin symme- 
try between the proton and the neutron, while sea quarks do not; then it can be found that

$$
R_{\text {sea }}=\frac{50\left(\varepsilon_{1}-\varepsilon_{0}\right)\left(\bar{q}^{p}-\bar{q}^{n}\right)}{3\left(u_{V}+d_{V}\right)} .
$$

From Eqs. (2.1) and (2.2) we know the relation

$$
\int_{0}^{1}\left[\bar{q}^{p}(x)-\bar{q}^{n}(x)\right]=\frac{3}{5} \int_{0}^{1}[\bar{u}(x)-\bar{d}(x)]
$$

between $p$ - $n$ isospin symmetry breaking and an asymmetric $\mathrm{SU}(2)$ sea; thus we can reasonably assume

$$
\left[\bar{q}^{p}(x)-\bar{q}^{n}(x)\right]=\frac{3}{5}[\bar{u}(x)-\bar{d}(x)] .
$$

Substituting this relation into Eq. (3.5) we arrive at Eq. (3.4). Thus the two explanations give approximately the same result for Eq. (3.2) introduced by Kumano and Londergan.

\section{B. Drell-Yan processes by proton scattering}

We now turn our attention to the Drell-Yan processes for protons scattered from proton and deuteron targets, which are discussed by Ellis and Stirling [19], and also by Kumano and Londergan [21] recently. The cross section of the process $p N \rightarrow l^{+} l^{-} X$ is sensitive to the seaantiquark distributions:

$s \frac{d^{2} \sigma^{p N}}{d \sqrt{\tau} d y}=\frac{8 \pi \alpha^{2}}{9 \sqrt{\tau}} \sum_{i} e_{i}^{2} q_{i}^{p}\left(x_{1}, M\right) \bar{q}_{i}^{N}\left(x_{2}, M\right)+[1 \leftrightarrow 2]$, where $\tau=M^{2} / s, x_{1}=\sqrt{\tau} e^{y}$, and $x_{2}=\sqrt{\tau} e^{-y}$. We ignore, following Ellis and Stirling and Kumano and Londergan, the contributions from strange and charm quarks and retain only the dominant "valence-sea" annihilation terms in the remainder; then we get, at $y=0$,

$$
\begin{gathered}
\sigma^{p p} \equiv s \frac{d^{2} \sigma^{p p}}{d \sqrt{\tau} d y}=\frac{8 \pi \alpha^{2}}{9 \sqrt{\tau}}\left(\frac{8}{9} u_{V}^{p} \bar{u}^{p}+\frac{2}{9} d_{V}^{p} \bar{d}^{p}\right), \\
\sigma^{p n} \equiv s \frac{d^{2} \sigma^{p n}}{d \sqrt{\tau} d y}=\frac{8 \pi \alpha^{2}}{9 \sqrt{\tau}}\left[\begin{array}{l}
\frac{4}{9}\left(u_{V}^{p} \bar{u}^{n}+u_{V}^{n} \bar{u}^{p}\right) \\
\left.+\frac{1}{9}\left(d_{V}^{p} \bar{d}^{n}+d_{V}^{n} \bar{d}^{p}\right)\right] .
\end{array}\right.
\end{gathered}
$$

The $p$ - $n$ cross-section asymmetry

$$
A_{\mathrm{DY}}=\frac{\sigma^{p p}-\sigma^{p n}}{\sigma^{p p}+\sigma^{p n}}
$$

discussed by Ellis-Stirling becomes

$$
A_{\mathrm{DY}}=\frac{\left(4 u_{V}-d_{V}\right)(\bar{u}-\bar{d})+\left(u_{V}-d_{V}\right)(4 \bar{u}-\bar{d})}{\left(4 u_{V}+d_{V}\right)(\bar{u}+\bar{d})+\left(u_{V}+d_{V}\right)(4 \bar{u}+\bar{d})}
$$

for a $\mathrm{SU}(2)$-asymmetric sea. It was found by Ellis and Stirling that the quantity Eq. (3.11) can change sign for an asymmetric sea, whereas it is positive in the case that the violation of the GSR is due to the unjustified $x \rightarrow$ 0 extrapolation of the data. We assume instead that only the valence quarks preserve the isospin symmetry between the proton and the neutron, while sea quarks do not. Thus we find

$$
A_{\mathrm{DY}}=\frac{\left(4 u_{V}-d_{V}\right) 5\left(\bar{q}^{p}-\bar{q}^{n}\right) / 3+\left(u_{V}-d_{V}\right)\left(\bar{q}^{p}+8 \bar{q}^{n}\right) / 3}{9\left(\sigma^{p p}+\sigma^{p n}\right)}
$$

Comparing Eq. (3.12) with Eq. (3.11), one can find that the $p$ - $n$ cross-section asymmetry could also change sign in the case of isospin symmetry breaking between the proton and the neutron. Using Eq. (3.7) and adopting $(4 \bar{u}-\bar{d}) \sim\left(\bar{q}^{p}+8 \bar{q}^{n}\right) / 3$, we see that Eq. (3.12) will give approximately the same value as Eq. (3.11). Therefore, the Drell-Yan processes discussed in the literature $[18$, $19,21]$ are not sufficient to distinguish between an asymmetric SU(2) sea and $p-n$ isospin symmetry breaking for charged pion scattering Eq. (3.2) as well as for proton scattering Eq. (3.10).

\section{SEMI-INCLUSIVE DEEP INELASTIC SCATTERING}

It has been suggested by Levelt, Mulders, and Schreiber (LMS) [20] that the semi-inclusive leptoproduction of hadrons in deep inelastic electron or muon scattering can be used as a check of SU(2) asymmetry in the proton sea. We will show that the breakdown of $p-n$ isospin symmetry could also contribute to this process and account for the data. According to the quark-parton model considerations [23], the number of hadrons $h$ produced by a scattering off the nucleon $N$ in a given bin of $x$ Bjorken variable and $z=E_{h} / \nu$ should be, up to a constant factor,

$$
N^{N h}(x, z) \equiv \sum_{i} e_{i}^{2} q_{i}^{N}(x) D_{i}^{h}(z),
$$

where $q_{i}^{N}(x)$ is the distribution function for quarks of flavor $i$ in the nucleon $N$ and $D_{i}^{h}$ is the fragmentation function of a quark with flavor $i$ into the hadron $h$ with energy $E_{h}=z \nu$. Defining

$$
Q(z)=\frac{N^{p+}-N^{n+}+N^{p-}-N^{n-}}{N^{p+}-N^{n+}-N^{p-}+N^{n-}},
$$

where $N^{N \pm}=\int d x N^{N \pm}(x, z)$ is for \pm charged hadrons, LMS found, assuming $p-n$ isospin symmetry [i.e., Eq. (3.3)],

$Q(z)=3 S_{G} \frac{4\left[D_{u}^{+}(z)+D_{\bar{u}}^{+}(z)\right]-\left[D_{d}^{+}(z)+D_{\bar{d}}^{+}(z)\right]}{4\left[D_{u}^{+}(z)-D_{\bar{u}}^{+}(z)\right]-\left[D_{d}^{+}(z)-D_{\bar{d}}^{+}(z)\right]}$,

which is directly proportional to the outcome of the GSR in inclusive leptoproduction. After some detailed examination of charged pions, kaons, and protons and antiprotons, LMS derived an expression for $Q^{\text {ch }}(z)$, using $D_{u}^{+}=D_{u}^{\pi+}+D_{u}^{K+}+D_{u}^{p}$ :

$$
z Q^{\mathrm{ch}}(z)=3 S_{G} z \frac{0.50 z^{2}+3.1 z+7.6}{3.2 z^{2}+11 z+0.84} .
$$


They found that the data are consistent with both $S_{G}=$ 0.24 and $S_{G}=1 / 3$.

We now examine the case that the violation of GSR is due to isospin symmetry breaking between the proton and the neutron, while preserving $\mathrm{SU}(2)$ symmetry in the sea of nucleons. It can be seen, from Eq. (4.1),

$$
\begin{aligned}
N^{p \pm}= & \frac{4}{9} u^{p} D_{u}^{ \pm}+\frac{4}{9} \bar{u}^{p} D_{\bar{u}}^{ \pm}+\frac{1}{9} d^{p} D_{d}^{ \pm} \\
& +\frac{1}{9} \bar{d}^{p} D_{\bar{d}}^{ \pm}+\frac{1}{9} s^{p} D_{s}^{ \pm}+\frac{1}{9} \bar{s}^{p} D_{\bar{s}}^{ \pm}, \\
N^{n \pm}= & \frac{4}{9} u^{n} D_{u}^{ \pm}+\frac{4}{9} \bar{u}^{n} D_{\bar{u}}^{ \pm}+\frac{1}{9} d^{n} D_{d}^{ \pm} \\
& +\frac{1}{9} \bar{d}^{n} D_{\bar{d}}^{ \pm}+\frac{1}{9} s^{n} D_{s}^{ \pm}+\frac{1}{9} \bar{s}^{n} D_{\bar{s}}^{ \pm} .
\end{aligned}
$$

$$
Q(z)=\frac{4\left[D_{u}^{+}(z)+D_{\bar{u}}^{+}(z)\right](1-2 \delta \bar{q})-\left[D_{d}^{+}(z)+D_{\bar{d}}^{+}(z)\right](1+2 \delta \bar{q})}{4\left[D_{u}^{+}(z)-D_{\bar{u}}^{+}(z)\right]-\left[D_{d}^{+}(z)-D_{\bar{d}}^{+}(z)\right]}
$$

where $\delta \bar{q}=\bar{q}^{n}-\bar{q}^{p}$ is the excess of sea quarks in the neutron over those in the proton. It can be seen that Eq. (4.7) is different from Eq. (4.3) unless $S_{G}=1 / 3$ [i.e., $\bar{d}-\bar{u}=0$ in the $\mathrm{SU}(2)$ sea asymmetry explanation and $\delta \bar{q}=\bar{q}^{n}-\bar{q}^{p}=0$ in the $p-n$ isospin symmetry-breaking explanation]. Following the similar analyses of Ref. [20] we find that the expressions $Q^{\pi}(z)$ and $Q^{p}(z)$ are the same as for an asymmetric $\mathrm{SU}(2)$. However, $Q^{K}(z)$ is now

$$
Q^{K}(z)=\frac{\left(D^{K}+\tilde{D}^{K}\right)\left(\frac{2}{5}+\frac{9}{5} S_{G}\right)-\frac{1}{2} \tilde{D}^{\prime K}\left(\frac{8}{5}-\frac{9}{5} S_{G}\right)}{D^{K}-\tilde{D}^{K}}
$$

which is different from Eq. (24) of Ref. [20] unless $S_{G}=$ $1 / 3$. Thus we have, for $D_{u}^{+}=D_{u}^{\pi+}+D_{u}^{K+}+D_{u}^{p}$,

$$
z Q^{\mathrm{ch}}(z)=3 S_{G} z \frac{0.80 z^{2}+3.37 z+7.63}{3.2 z^{2}+11 z+0.84}
$$

for $S_{G}=0.24$ in the case of isospin symmetry breaking between the proton and the neutron. Comparing Eq. (4.4) and Eq. (4.9), we see that the expressions for $Q^{\mathrm{ch}}(z)$ are different for the two explanations. In the latter case the result is between the GSR prediction and the prediction for an asymmetric $\mathrm{SU}(2)$ sea and thus is also consistent with the data of Ref. [20]. To distinguish between an asymmetric SU(2) sea and $p-n$ isospin symmetry breaking from $Q^{\text {ch }}(z)$, we need data with better accuracy.
Assuming charge conjugation invariance one has $D_{u}^{ \pm}=$ $D_{\bar{u}}^{\mp}$ and $D_{\bar{d}}^{ \pm}=D_{\bar{d}}^{\mp}$. The flavor number conservation requires $u_{V}=u^{p}-\bar{u}^{p}=d^{n}-\bar{d}^{n}=2$ and $d_{V}=d^{p}-\bar{d}^{p}=$ $u^{n}-\bar{u}^{n}=1$; thus, one obtains

$$
\begin{aligned}
N^{p+}-N^{n+}= & \frac{4}{9} D_{u}^{+}(1-\delta \bar{q})-\frac{4}{9} D_{\bar{u}}^{+} \delta \bar{q} \\
& -\frac{1}{9} D_{d}^{+}(1+\delta \bar{q})-\frac{1}{9} D_{\bar{d}}^{+} \delta \bar{q}, \\
N^{p-}-N^{n-}= & \frac{4}{9} D_{\bar{u}}^{+}(1-\delta \bar{q})-\frac{4}{9} D_{u}^{+} \delta \bar{q} \\
& -\frac{1}{9} D_{\bar{d}}^{+}(1+\delta \bar{q})-\frac{1}{9} D_{d}^{+} \delta \bar{q},
\end{aligned}
$$

from which we get 
[6] A. Signal, A.W. Schreiber, and A.W. Thomas, Mod. Phys. Lett. A 6, 271 (1991).

[7] J. Stern and G. Clément, Phys. Lett. B 264, 426 (1991).

[8] W-Y.P. Hwang, J. Speth, and G.E. Brown, Z. Phys. A 339, 383 (1991); Phys. Rev. D 46, 1198 (1992).

[9] W. Melnitchouk, A.W. Thomas, and A.I. Signal, Z. Phys. A 340, 85 (1991).

[10] E.J. Eichten, I. Hinchliffe, and C. Quigg, Phys. Rev. D 45, 2269 (1992).

[11] M. Wakamatsu, Phys. Lett. B 269, 394 (1991).

[12] A.D. Martin, W.J. Stirling, and R.G. Roberts, Phys. Lett. B 252, 653 (1990).

[13] M. Anselmino and E. Predazzi, Phys. Lett. B 254, 203 (1991). See, however, M. Anselmino, V. Barone, F. Caruso, and E. Predazzi, Z. Phys. C 55, 97 (1992), for a revision of the result.

[14] B.-Q. Ma, Phys. Lett. B 274, 111 (1992). In this paper "[13]" in line 17 of the first paragraph should be "[12]," and "0.84" in Eq. (12) should be "0.084."

[15] L.P. Kaptari and A.Yu. Umnikov, Phys. Lett. B 272, 359 (1991); 259, 155 (1991).

[16] L.N. Epele, H. Fanchiotti, C.A. Carcía Canal, and R. Sassot, Phys. Lett. B 275, 155 (1992).

[17] V.R. Zoller, Phys. Lett. B 279, 145 (1992).

[18] S. Kumano and J.T. Londergan, Phys. Rev. D 44, 717 (1991)

[19] S.D. Ellis and W.J. Stirling, Phys. Lett. B 256, 258 (1991).

[20] J. Levelt, P.J. Mulders, and A.W. Schreiber, Phys. Lett. B 263, 498 (1991).

[21] S. Kumano and J.T. Londergan, Phys. Rev. D 46, 457 (1992).

[22] R.D. Field and R.P. Feynman, Phys. Rev. D 15, 2590 (1977)

[23] See, e.g., F.E. Close, An Introduction to Quarks and Partons (Academic, London, 1979), p. 245. 